MATHEMATICS OF COMPUTATION

229 , Pages $65-82$

S 0025-5718(99)01076-5

Article electronically published on March 2, 1999

\title{
A DOMAIN EMBEDDING PRECONDITIONER FOR THE LAGRANGE MULTIPLIER SYSTEM
}

\author{
EINAR HAUG AND RAGNAR WINTHER
}

\begin{abstract}
Finite element approximations for the Dirichlet problem associated to a second-order elliptic differential equation are studied. The purpose of this paper is to discuss domain embedding preconditioners for discrete systems. The essential boundary condition on the interior interface is removed by introducing Lagrange multipliers. The associated discrete system, with a saddle point structure, is preconditioned by a block diagonal preconditioner. The main contribution of this paper is to propose a new operator, constructed from the $\boldsymbol{H}$ (div)-inner product, for the block of the preconditioner corresponding to the multipliers.
\end{abstract}

\section{INTRODUCTION}

The purpose of this paper is to discuss domain embedding preconditioners for second-order elliptic equations with Dirichlet boundary conditions. Hence, if the geometry of the domain $\Omega$ is complex, or irregular, we utilize an embedding of $\Omega$ into an extended domain $\Omega_{e}$ in order to construct a preconditioner defined on $\Omega$.

As a model problem we will consider

$$
\begin{array}{rlrl}
-\operatorname{div}(K(\boldsymbol{x}) \operatorname{grad} p) & =f & & \text { in } \Omega, \\
p=g & & \text { on } \Gamma, \\
p=0 & & \text { on } \partial \Omega \backslash \Gamma .
\end{array}
$$

Here, $\Omega \subset \mathbb{R}^{2}$ is a bounded polygonal domain and $\partial \Omega$ is the boundary. The part of the boundary that will become an interior curve in the extended domain $\Omega_{e}$ (i.e. the interface between $\Omega$ and $\Omega_{e} \backslash \Omega$ ) is denoted by $\Gamma$. For simplicity, we assume that $\partial \Omega \backslash \Gamma$ is nonempty and connected. However, the results will also hold for a union of connected curves, as illustrated in Figure 1. The coefficient matrix $K(\boldsymbol{x})$ is assumed to be bounded, symmetric and uniformly positive definite on the extended domain $\Omega_{e}$.

A standard finite element discretization of the boundary value problem (1.1) leads to a discrete, symmetric and positive definite system of the form

$$
A_{h} p_{h}=f_{h},
$$

where $h>0$ is a small discretization parameter indicating the mesh size. The condition number of the coefficient operator $A_{h}$ will increase with decreasing mesh

Received by the editor March 26, 1997 and, in revised form, March 17, 1998.

1991 Mathematics Subject Classification. Primary 65F10, 65N22, 65N30.

Key words and phrases. Second-order elliptic problems, Dirichlet boundary conditions, Lagrange multiplier method, preconditioning, domain embedding.

This work was partially supported by the Research Council of Norway (NFR), program no. 100998/420 and STP.29643. 

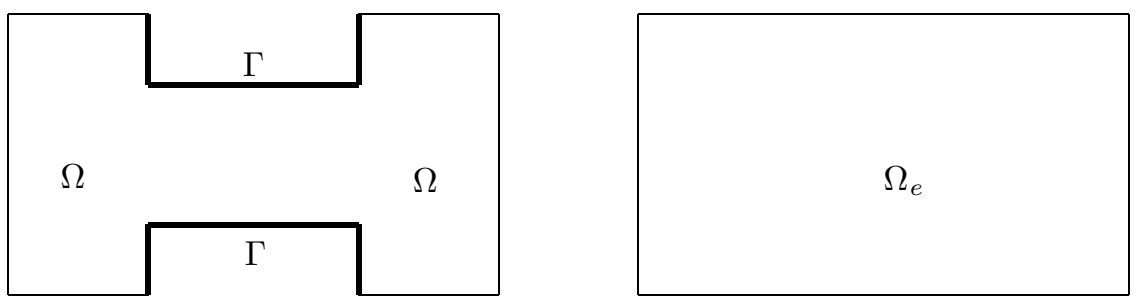

Figure 1. Left: An example of the domain $\Omega$ where the $\Gamma$-part of the boundary is drawn in bold. Right: The extended domain $\Omega_{e}$.

size like $O\left(h^{-2}\right)$. Therefore, in order to obtain effective iterative methods for the discrete system, the construction of a suitable preconditioner $B_{h}$ is necessary.

Multigrid methods will for example generate very effective preconditioners $B_{h}$. However, on more complex domains the construction of a suitable scale of spaces necessary for defining these operators might represent a nontrivial practical problem. In a domain embedding approach we try to overcome this difficulty by utilizing a corresponding preconditioner $B_{e, h}$ defined on the extended, and more regular, domain $\Omega_{e}$. If we need to solve the problem (1.1) for a sequence of domains $\Omega$ which all can be embedded in a fixed domain $\Omega_{e}$, this approach seems rather attractive. Such computations may for example occur in shape optimization problems or in the computation of flow around a moving rigid body (cf. Glowinski, Pan and Périaux [11]).

A rather obvious approach is to consider operators $B_{h}$ of the form

$$
B_{h}=R_{h} B_{e, h} E_{h},
$$

where $E_{h}$ and $R_{h}$ are proper extension and restriction operators, respectively. If we consider the problem (1.1), but with the Dirichlet boundary conditions on $\Gamma$ replaced by natural boundary conditions, then suitable operators of the form (1.3) can easily be constructed. If $E_{h}$ is essentially chosen as the extension by zero operator and $R_{h}$ as the restriction operator, then the operator $B_{h}$, constructed by (1.3), will be a uniform preconditioner for $A_{h}$, under the assumption that $B_{e, h}$ has the corresponding property on $\Omega_{e}$. We refer to Astrakhantsev [3] and Marchuk, Kuznetsov and Matsokin [13] for a discussion of these results.

However, the situation is not as straightforward for essential boundary conditions on $\Gamma$. An approach utilizing approximate harmonic extension operators has been studied by Nepomnyaschikh [14], [15] and [16], while Vassilevski [20] has proposed an alternative extension operator based on a wavelet-like hierarchical decomposition of finite element spaces.

One possible approach to constructing domain embedding preconditioners for the Dirichlet problem is to change the weak formulation of the problem in such a way that the Dirichlet boundary conditions become natural. This is for example achieved by the so-called mixed formulation. In fact, domain embedding preconditioners for mixed finite element approximations of the Dirichlet problem were constructed by Rusten, Vassilevski and Winther [19].

As an alternative to this, in this paper we shall discuss the Lagrange multiplier method introduced by Babuška [4] (cf. also Bramble [5]). In this method the essential boundary conditions are transformed to constraints in a larger function space. 
The advantage of this approach, compared to the mixed method, is that we are essentially still using the standard finite element method. However, the Dirichlet boundary conditions are relaxed and incorporated into the discrete system. The effect is that the exact numerical solution of the Lagrange multiplier system is the same as for the standard finite element method, but the sequence of approximations generated by an iterative method will only satisfy the boundary conditions approximately.

In the Lagrange multiplier method the positive definite system (1.2) is replaced by an indefinite system with a typical saddle point structure. This system will be preconditioned by a block diagonal preconditioner, where the first block, $M_{h}$, corresponds to a preconditioner for the Neumann problem. The second block of the preconditioner, $N_{h}$, is a boundary operator defined on $\Gamma$. In this respect our approach is closely related to the discussion in Rossi [18] (cf. also Glowinski, Pan and Périaux [10]). In [18] the preconditioner $N_{h}$ is constructed directly on the interface $\Gamma$. The main purpose of this paper is to discuss a new strategy for constructing a proper boundary operator $N_{h}$. We propose to construct this operator from a preconditioner of the $\boldsymbol{H}(\operatorname{div})$-inner product on the extended domain $\Omega_{e}$. This global $\boldsymbol{H}$ (div)-preconditioner is defined independently of the interface $\Gamma$. The interface will only enter into the right hand side of the system defining the operator $N_{h}$. The $\boldsymbol{H}(\mathrm{div})$-preconditioner on $\Omega_{e}$ will be of the form proposed in Arnold, Falk and Winther [2].

The outline of this paper is as follows: In $\S 2$ we formulate the problem using Lagrange multipliers and identify the proper operators and function spaces. In $\S 3$ we discuss a possible preconditioner for the continuous system. In $\S 4$ we introduce the appropriate finite element spaces and the corresponding Lagrange multiplier method. Inspired by the discussion in $\S 3$, we then derive the preconditioner for the discrete system. Finally, in $\S 5$ we report some numerical experiments based on the elliptic problem (1.1). We also present computations done for the Stokes problem with Dirichlet boundary conditions.

\section{Preliminaries}

The inner product on $L^{2}(\Omega)$ will be denoted by $(\cdot, \cdot)$, and the $L^{2}$-inner product on $\Gamma$ by $\langle\cdot, \cdot\rangle$. The same notation will also be used for vector valued functions. Corresponding bold symbols are used for vector valued functions, operators and function spaces.

The Sobolev space consisting of functions with first order partial derivatives in $L^{2}(\Omega)$ will be denoted $H^{1}(\Omega)$, while the subspaces $H_{0}^{1}(\Omega)$ and $H_{0}^{1}(\Omega ; \Gamma)$ are defined by

$$
\begin{aligned}
H_{0}^{1}(\Omega) & =\left\{q \in H^{1}(\Omega):\left.q\right|_{\partial \Omega}=0\right\}, \\
H_{0}^{1}(\Omega ; \Gamma) & =\left\{q \in H^{1}(\Omega):\left.q\right|_{\partial \Omega \backslash \Gamma}=0\right\} .
\end{aligned}
$$

The space $H_{0}^{1 / 2}(\Gamma)$ is given by

$$
H_{0}^{1 / 2}(\Gamma)=\left\{\left.q\right|_{\Gamma}: q \in H_{0}^{1}(\Omega ; \Gamma)\right\} .
$$

This space can equivalently be characterized as the interpolation space half way between $L^{2}(\Gamma)$ and $H_{0}^{1}(\Gamma)$, where the subscript zero indicates that the functions 
are zero at the endpoints of $\Gamma$. The norm on $H_{0}^{1 / 2}(\Gamma)$ is

$$
|g|_{1 / 2}=\inf _{q \in H_{0}^{1}(\Omega ; \Gamma)}\left\{\|q\|_{1}:\left.q\right|_{\Gamma}=g\right\},
$$

where $\|\cdot\|_{1}$ is the usual $H^{1}$-norm. The spaces $H_{0}^{1}(\Omega ; \Gamma)^{*}$ and $H^{-1 / 2}(\Gamma)=H_{0}^{1 / 2}(\Gamma)^{*}$ are the corresponding dual spaces with respect to the proper $L^{2}$-inner products. The norms on these spaces are

$$
\|f\|_{-1}=\sup _{q \in H_{0}^{1}(\Omega ; \Gamma)} \frac{(f, q)}{\|q\|_{1}} \quad \text { and } \quad|\sigma|_{-1 / 2}=\sup _{g \in H_{0}^{1 / 2}(\Gamma)} \frac{\langle\sigma, g\rangle}{|g|_{1 / 2}} .
$$

Hence, the duality pairing between $H_{0}^{1}(\Omega ; \Gamma)$ and $H_{0}^{1}(\Omega ; \Gamma)^{*}$ is an extension of the $L^{2}$-inner product $(\cdot, \cdot)$, which still will be denoted $(\cdot, \cdot)$. A similar remark applies to the inner product $\langle\cdot, \cdot\rangle$.

The bilinear form associated with the differential operator in (1.1) is

$$
a(p, q)=\int_{\Omega} K(\boldsymbol{x}) \operatorname{grad} p \cdot \operatorname{grad} q d \boldsymbol{x} \quad \text { for } p, q \in H^{1}(\Omega) .
$$

The standard weak formulation of the problem (1.1) is then to find $p \in H_{0}^{1}(\Omega ; \Gamma)$ with $\left.p\right|_{\Gamma}=g$ such that

$$
a(p, q)=(f, q) \quad \text { for all } q \in H_{0}^{1}(\Omega) .
$$

This is equivalent to minimizing the functional

$$
\frac{1}{2} a(p, p)-(f, p) \text { over }\left\{q \in H_{0}^{1}(\Omega ; \Gamma):\left.q\right|_{\Gamma}=g\right\} .
$$

The Lagrange multiplier formulation [4] is derived by reformulating this problem as a constrained minimization problem over the larger space $H_{0}^{1}(\Omega ; \Gamma)$. This leads to a linear saddle point problem of the form: For $f \in H_{0}^{1}(\Omega ; \Gamma)^{*}$ and $g \in H_{0}^{1 / 2}(\Gamma)$, find $(p, \lambda) \in H_{0}^{1}(\Omega ; \Gamma) \times H^{-1 / 2}(\Gamma)$ such that

$$
\begin{aligned}
& a(p, q)+\left\langle\lambda,\left.q\right|_{\Gamma}\right\rangle=(f, q) \quad \text { for all } q \in H_{0}^{1}(\Omega ; \Gamma) \text {, } \\
& \left\langle\left. p\right|_{\Gamma}, \sigma\right\rangle \quad=\langle g, \sigma\rangle \quad \text { for all } \sigma \in H^{-1 / 2}(\Gamma) \text {. }
\end{aligned}
$$

Of course, the solution $p$ will be the same for both problems (2.4) and (2.5). Furthermore, the Lagrange multiplier $\lambda$ equals $-\partial p / \partial \boldsymbol{n}$ on $\Gamma$, where $\boldsymbol{n}$ is the unit outward normal vector on $\Gamma$.

In the weak formulation (2.5) the essential boundary condition $\left.p\right|_{\Gamma}=g$ has been transformed to a natural boundary condition. This is a key observation for the construction of domain embedding preconditioners. However, while (2.4) is a positive definite problem, the problem (2.5) is symmetric, but indefinite.

The system (2.5) can alternatively be written in operator form as

$$
\mathcal{A}\left(\begin{array}{l}
p \\
\lambda
\end{array}\right)=\left(\begin{array}{l}
f \\
g
\end{array}\right),
$$

where the coefficient operator $\mathcal{A}$ is defined by

$$
\mathcal{A}=\left(\begin{array}{cc}
A & T^{*} \\
T & 0
\end{array}\right) .
$$

Here, $A: H_{0}^{1}(\Omega ; \Gamma) \mapsto H_{0}^{1}(\Omega ; \Gamma)^{*}$ is given by

$$
(A p, q)=a(p, q) \quad \text { for } p, q \in H_{0}^{1}(\Omega ; \Gamma),
$$


while the trace operator $T: H_{0}^{1}(\Omega ; \Gamma) \mapsto H_{0}^{1 / 2}(\Gamma)$ and its dual $T^{*}: H^{-1 / 2}(\Gamma) \mapsto$ $H_{0}^{1}(\Omega ; \Gamma)^{*}$ are given by

$$
\langle T p, \lambda\rangle=\left\langle\left. p\right|_{\Gamma}, \lambda\right\rangle=\left(p, T^{*} \lambda\right) \quad \text { for } \lambda \in H^{-1 / 2}(\Gamma) \text { and } p \in H_{0}^{1}(\Omega ; \Gamma) .
$$

The operator $\mathcal{A}$ maps the product space $X=H_{0}^{1}(\Omega ; \Gamma) \times H^{-1 / 2}(\Gamma)$ into its dual space $X^{*}=H_{0}^{1}(\Omega ; \Gamma)^{*} \times H_{0}^{1 / 2}(\Gamma)$. Furthermore, $\mathcal{A}$ is an indefinite operator which is symmetric with respect to the duality pairing between $X$ and $X^{*}$. The spaces $X$ and $X^{*}$ are equipped with the norms

$$
\left\|\left(\begin{array}{c}
p \\
\lambda
\end{array}\right)\right\|_{X}^{2}=\|p\|_{1}^{2}+|\lambda|_{-1 / 2}^{2} \quad \text { and } \quad\left\|\left(\begin{array}{c}
f \\
g
\end{array}\right)\right\|_{X^{*}}^{2}=\|f\|_{-1}^{2}+|g|_{1 / 2}^{2} .
$$

Since $A$ is continuous and coercive, and $T$ is continuous and satisfies the BabuškaBrezzi condition

$$
\sup _{q \in H_{0}^{1}(\Omega ; \Gamma)} \frac{\langle T q, \sigma\rangle}{\|q\|_{1}} \geq \alpha|\sigma|_{-1 / 2} \quad \text { for all } \sigma \in H^{-1 / 2}(\Gamma) \text { with } \alpha>0,
$$

it follows from the general theory discussed in [7] that the coefficient operator $\mathcal{A}$ is an isomorphism from $X$ into $X^{*}$. Hence, there exists a unique and stable solution of the problem (2.5).

\section{MAPPING PROPERTIES AND DOMAIN EMBEDDING PRECONDITIONERS}

The main purpose of this paper is to discuss block diagonal preconditioners for discrete versions of the Lagrange multiplier system (2.5). However, in order to motivate our choice of preconditioners we will first discuss how to "precondition" the continuous system.

We refer to an operator $\mathcal{B}$ as a preconditioner for $\mathcal{A}$ if $\mathcal{B}: X^{*} \mapsto X$ is an isomorphism which is symmetric and positive definite as a map from $X^{*}$ to $X$, i.e.

$$
(\mathcal{B} \ell, \ell) \geq \alpha\|\ell\|_{X^{*}}^{2} \quad \text { for all } \ell \in X^{*}
$$

where $\alpha>0$ and $(\cdot, \cdot)$ denotes the duality pairing between $X$ and $X^{*}$. Hence, the system (2.6) is equivalent to the system

$$
\mathcal{B A}\left(\begin{array}{l}
p \\
\lambda
\end{array}\right)=\mathcal{B}\left(\begin{array}{l}
f \\
g
\end{array}\right)
$$

We observe that the coefficient operator $\mathcal{B A}$ in (3.1) is an isomorphism mapping $X$ into itself, i.e.

$$
\|\mathcal{B} \mathcal{A}\|_{\mathcal{L}(X, X)} \quad \text { and } \quad\left\|(\mathcal{B A})^{-1}\right\|_{\mathcal{L}(X, X)} \quad \text { are bounded. }
$$

Furthermore, $\left(\mathcal{B}^{-1},, \cdot\right)$ is a new inner product on $X$ which is equivalent to the one introduced above. The coefficient operator $\mathcal{B A}$ is symmetric with respect to this inner product.

A preconditioned differential system of the form (3.1) can, in theory, be solved by a Krylov space method like the minimum residual method (cf. [12] or [17]) or the conjugate gradient method applied to the normal equations. The method is well defined as long as the coefficient operator $\mathcal{B A}$ maps $X$ into itself, and it is guaranteed to converge in the norm of $X$ if the spectral condition number

$$
\kappa(\mathcal{B A})=\|\mathcal{B} \mathcal{A}\|_{\mathcal{L}(X, X)}\left\|(\mathcal{B A})^{-1}\right\|_{\mathcal{L}(X, X)}
$$

is finite. Hence, property (3.2) ensures convergence. 
Since $X$ is a product space in our case, it is natural to consider a block diagonal preconditioner $\mathcal{B}: X^{*} \mapsto X$ of the form

$$
\mathcal{B}=\left(\begin{array}{cc}
M & 0 \\
0 & N
\end{array}\right)
$$

The required mapping properties of $\mathcal{B}$ then imply that $M: H_{0}^{1}(\Omega ; \Gamma)^{*} \mapsto H_{0}^{1}(\Omega ; \Gamma)$ and $N: H_{0}^{1 / 2}(\Gamma) \mapsto H^{-1 / 2}(\Gamma)$ should be chosen as symmetric, positive definite isomorphisms.

Of course, in practical computations the system (2.6) will be approximated by a corresponding discrete system, with coefficient operator $\mathcal{A}_{h}$. And as a consequence the preconditioner $\mathcal{B}$ will be replaced by a discrete operator $\mathcal{B}_{h}$ of the form

$$
\mathcal{B}_{h}=\left(\begin{array}{cc}
M_{h} & 0 \\
0 & N_{h}
\end{array}\right) \text {. }
$$

Discrete versions of the mapping requirements for $M$ and $N$ specified above will then guarantee that the spectral condition number of $\mathcal{B}_{h} \mathcal{A}_{h}$ is independent of the discretization parameter $h$. In order to define an effective iterative method it is also necessary that $M_{h}$ and $N_{h}$ are easy to evaluate.

In the next section we shall discuss how we can utilize domain embedding in order to construct suitable operators $M_{h}$ and $N_{h}$. However, we will first present the continuous versions of these operators.

3.1. The construction of $\boldsymbol{M}$. Let $E: H_{0}^{1}(\Omega ; \Gamma)^{*} \mapsto H_{0}^{1}\left(\Omega_{e}\right)^{*}=H^{-1}\left(\Omega_{e}\right)$ be the extension by zero operator and $R: H_{0}^{1}\left(\Omega_{e}\right) \mapsto H_{0}^{1}(\Omega ; \Gamma)$ the restriction operator. Then $E$ and $R$ are dual operators. Furthermore, if $B_{e}: H^{-1}\left(\Omega_{e}\right) \mapsto H_{0}^{1}\left(\Omega_{e}\right)$ is an isomorphism then it can be seen that

$$
M=R B_{e} E: H_{0}^{1}(\Omega ; \Gamma)^{*} \mapsto H_{0}^{1}(\Omega ; \Gamma)
$$

is an isomorphism. Hence, the operator $M$, constructed by utilizing domain embedding, has the proper mapping property derived above.

In the discrete case the operator $B_{e}$ will correspond to a preconditioner for a Dirichlet problem for a second-order elliptic problem on the extended domain $\Omega_{e}$. The construction of the operator $M$ corresponds to the standard domain embedding preconditioner for the Neumann problem (cf., e.g., [3] or [13]). The desired mapping property of the operator $M$, inherited from $B_{e}$, is a consequence of the so-called "fictitious space lemma", given in [14]. In fact, it is the possible use of this operator that is the main motivation for introducing the Lagrange multiplier formulation in order to utilize domain embedding for Dirichlet problems.

3.2. The construction of $\boldsymbol{N}$. The construction of the boundary preconditioner $N: H_{0}^{1 / 2}(\Gamma) \mapsto H^{-1 / 2}(\Gamma)$ is not as obvious. A seemingly natural choice would be the following:

$$
N g=\left.\frac{\partial \phi}{\partial \boldsymbol{n}}\right|_{\Gamma}
$$

where $\boldsymbol{n}$ is the outward unit vector on $\Gamma$ and the $H^{1}$-function $\phi$ is the solution of

$$
-\operatorname{div}(\operatorname{grad} \phi)=0 \quad \text { in } \Omega, \quad \text { and } \phi= \begin{cases}g & \text { on } \Gamma, \\ 0 & \text { on } \partial \Omega \backslash \Gamma .\end{cases}
$$

This operator has all the required properties, except that we have not utilized domain embedding. In fact, in order to evaluate $N$ we have to solve a Dirichlet 
problem on the original domain $\Omega$, and this is exactly what we want to avoid by using domain embedding.

Another possibility is to use some kind of boundary operator on $\Gamma$. We refer to [18] for this approach. As an alternative to this we propose an operator $N$ based on a preconditioner for the $\boldsymbol{H}\left(\operatorname{div} ; \Omega_{e}\right)$-inner product. Hence, in correspondence with the construction of the operator $M$ above, also the operator $N$ is defined from a suitable preconditioner with respect to the extended and regular domain $\Omega_{e}$.

The Hilbert space $\boldsymbol{H}\left(\operatorname{div} ; \Omega_{e}\right)$ consists of square-integrable vector fields with square-integrable divergence in the domain $\Omega_{e}$. The inner product $\Lambda(\cdot, \cdot)$ on $\boldsymbol{H}\left(\operatorname{div} ; \Omega_{e}\right)$ is defined by

$$
\Lambda(\boldsymbol{u}, \boldsymbol{v})=(\boldsymbol{u}, \boldsymbol{v})_{e}+(\operatorname{div} \boldsymbol{u}, \operatorname{div} \boldsymbol{v})_{e} \quad \text { for } \boldsymbol{u}, \boldsymbol{v} \in \boldsymbol{H}\left(\operatorname{div} ; \Omega_{e}\right),
$$

where $(\cdot, \cdot)_{e}$ is the inner product on $L^{2}\left(\Omega_{e}\right)$. The related linear operator $\boldsymbol{\Lambda}$ : $\boldsymbol{H}\left(\operatorname{div} ; \Omega_{e}\right) \mapsto \boldsymbol{H}\left(\operatorname{div} ; \Omega_{e}\right)^{*}$ is defined by

$$
(\boldsymbol{\Lambda} \boldsymbol{u}, \boldsymbol{v})_{e}=\Lambda(\boldsymbol{u}, \boldsymbol{v}) \quad \text { for } \boldsymbol{u}, \boldsymbol{v} \in \boldsymbol{H}\left(\operatorname{div} ; \Omega_{e}\right),
$$

or equivalently by

$$
\Lambda=I-\operatorname{grad} \operatorname{div} .
$$

Consider the following problem: For a given $g \in H_{0}^{1 / 2}(\Gamma)$ find $\boldsymbol{u} \in \boldsymbol{H}\left(\operatorname{div} ; \Omega_{e}\right)$ such that

$$
\Lambda(\boldsymbol{u}, \boldsymbol{v})=\left\langle g,(\boldsymbol{v} \cdot \boldsymbol{n})_{\Gamma}\right\rangle \quad \text { for all } \boldsymbol{v} \in \boldsymbol{H}\left(\operatorname{div} ; \Omega_{e}\right)
$$

The boundary value problem corresponding to (3.7) is

$$
\boldsymbol{u}-\operatorname{grad}(\operatorname{div} \boldsymbol{u})=\mathbf{0} \quad \text { in } \Omega_{e}, \quad \text { and } \quad\left\{\begin{array}{lll}
{[\operatorname{div} \boldsymbol{u}]=} & \text { on } \Gamma, \\
\operatorname{div} \boldsymbol{u}=0 & \text { on } \partial \Omega_{e} .
\end{array}\right.
$$

Here, $[\operatorname{div} \boldsymbol{u}]$ denotes the jump in $\operatorname{div} \boldsymbol{u}$ across $\Gamma$. The important difference between equation (3.8) and equation (3.4) is that $[\operatorname{div} \boldsymbol{u}]=g$ is imposed as a natural boundary condition in (3.8), while $g$ enters in an essential boundary condition in (3.4).

The suitable preconditioner $N: H_{0}^{1 / 2}(\Gamma) \mapsto H^{-1 / 2}(\Gamma)$ is given by

$$
N g=(\boldsymbol{u} \cdot \boldsymbol{n})_{\Gamma} \quad \text { for } g \in H_{0}^{1 / 2}(\Gamma)
$$

where $\boldsymbol{u} \in \boldsymbol{H}\left(\operatorname{div} ; \Omega_{e}\right)$ is the solution of (3.7).

If $\boldsymbol{v} \in \boldsymbol{H}\left(\operatorname{div} ; \Omega_{e}\right)$ then $(\boldsymbol{v} \cdot \boldsymbol{n})_{\Gamma}$ is continuous over the interface $\Gamma$, i.e. the normal component of $\boldsymbol{v}$ taken from the outside or inside of $\Gamma$ is the same. Furthermore, it follows essentially from the standard trace theorem for $\boldsymbol{H}$ (div) (cf. Theorem 2.5 of [9]) that

$$
\left|(\boldsymbol{v} \cdot \boldsymbol{n})_{\Gamma}\right|_{-1 / 2} \leq c_{1} \|\left.\boldsymbol{v}\right|_{\text {div }} \quad \text { for all } \boldsymbol{v} \in \boldsymbol{H}\left(\operatorname{div} ; \Omega_{e}\right),
$$

where $\|\boldsymbol{v}\|_{\text {div }}=\Lambda(\boldsymbol{v}, \boldsymbol{v})^{1 / 2}$ and $c_{1}>0$ is a constant independent of $\boldsymbol{v}$.

From this trace inequality it follows that $N: H_{0}^{1 / 2}(\Gamma) \mapsto H^{-1 / 2}(\Gamma)$ is welldefined and bounded. For if $g \in H_{0}^{1 / 2}(\Gamma)$ and $\boldsymbol{u}$ is the corresponding solution of (3.7), then

$$
c_{1}^{-2}\left|(\boldsymbol{u} \cdot \boldsymbol{n})_{\Gamma}\right|_{-1 / 2}^{2} \leq \Lambda(\boldsymbol{u}, \boldsymbol{u})=\left\langle g,(\boldsymbol{u} \cdot \boldsymbol{n})_{\Gamma}\right\rangle \leq|g|_{1 / 2}\left|(\boldsymbol{u} \cdot \boldsymbol{n})_{\Gamma}\right|_{-1 / 2},
$$

and therefore

$$
|N g|_{-1 / 2} \leq c_{1}^{2}|g|_{1 / 2}
$$


Hence, we have shown that $N: H_{0}^{1 / 2}(\Gamma) \mapsto H^{-1 / 2}(\Gamma)$ is bounded. However, the following theorem shows that $N$ has all the properties required for the preconditioner.

Theorem 3.1. The operator $N$, defined by (3.9), is a symmetric, positive definite isomorphism from $H_{0}^{1 / 2}(\Gamma)$ into $H^{-1 / 2}(\Gamma)$.

This theorem follows from well-known properties of $\boldsymbol{H}$ (div)-spaces, given for example in [9]. However, since we need to prove a discrete version of this result in the next section, let us recall the basic steps.

If $N g_{1}=\left(\boldsymbol{u}_{1} \cdot \boldsymbol{n}\right)_{\Gamma}$ and $N g_{2}=\left(\boldsymbol{u}_{2} \cdot \boldsymbol{n}\right)_{\Gamma}$, then the symmetry of $N$ follows from the identity

$$
\left\langle N g_{1}, g_{2}\right\rangle=\left\langle\left(\boldsymbol{u}_{1} \cdot \boldsymbol{n}\right)_{\Gamma}, g_{2}\right\rangle=\Lambda\left(\boldsymbol{u}_{1}, \boldsymbol{u}_{2}\right) .
$$

We have already shown that $N$ is bounded (equation (3.12)). That $N^{-1}$ is bounded, or equivalently that $g$ is bounded by $N g$, follows from the extension result, cf. [9]:

$$
\begin{aligned}
& \text { Given } \mu \in H^{-1 / 2}(\Gamma) \text {, there exists a } \boldsymbol{v} \in \boldsymbol{H}\left(\operatorname{div} ; \Omega_{e}\right) \text { with }(\boldsymbol{v} \cdot \boldsymbol{n})_{\Gamma}=\mu \\
& \text { and }\|\boldsymbol{v}\|_{\text {div }} \leq c|\mu|_{-1 / 2} \text { for some positive constant } c \text { independent of } \mu .
\end{aligned}
$$

Using this extension result, we obtain by duality that for any $g \in H_{0}^{1 / 2}(\Gamma)$

$$
\begin{aligned}
|g|_{1 / 2} & =\sup _{\mu \in H^{-1 / 2}(\Gamma)} \frac{\langle g, \mu\rangle}{|\mu|_{-1 / 2}} \leq c \sup _{\boldsymbol{v} \in \boldsymbol{H}\left(\operatorname{div} ; \Omega_{e}\right)} \frac{\left\langle g,(\boldsymbol{v} \cdot \boldsymbol{n})_{\Gamma}\right\rangle}{\|\boldsymbol{v}\|_{\text {div }}} \\
& =c \sup _{\boldsymbol{v} \in \boldsymbol{H}\left(\operatorname{div} ; \Omega_{e}\right)} \frac{\Lambda(\boldsymbol{u}, \boldsymbol{v})}{\|\boldsymbol{v}\|_{\text {div }}} \leq c\|\boldsymbol{u}\|_{\text {div }} \leq c|g|_{1 / 2}^{1 / 2}\left|(\boldsymbol{u} \cdot \boldsymbol{n})_{\Gamma}\right|_{-1 / 2}^{1 / 2}
\end{aligned}
$$

which implies that $N^{-1} \in \mathcal{L}\left(H^{-1 / 2}(\Gamma), H_{0}^{1 / 2}(\Gamma)\right)$. Finally, (3.11) and (3.14) imply that $N$ is positive definite.

In order to evaluate the operator $N$ we need to solve problem (3.7). Alternatively we may use an operator with the same mapping properties as $\boldsymbol{\Lambda}^{-1}$, i.e. a uniform preconditioner for $\boldsymbol{\Lambda}$. This subject will be further discussed for the discrete operator $\boldsymbol{\Lambda}_{h}$ in $\S 4.1$.

With the above choices for $M$ and $N$ the block diagonal operator $\mathcal{B}$ will be a symmetric and positive definite isomorphism, making it an acceptable choice for a preconditioner for the continuous problem. Our next task is then to find suitable discrete spaces and discrete operators $M_{h}$ and $N_{h}$. The requirements for the discrete operators are that they must have mapping properties which are discrete analogs of the requirements for their continuous counterpart. In addition they must be easy to evaluate.

\section{DisCRETIZATION}

The Lagrange multiplier method is derived from the weak formulation (2.5). We will use finite element spaces $W_{h} \subset H_{0}^{1}(\Omega ; \Gamma)$ and $S_{h} \subset H_{0}^{1 / 2}(\Gamma)$ based on a family of triangulations $\left\{\mathcal{T}_{h}\right\}_{h \in(0,1]}$ of $\Omega$, where $h$ denotes the characteristic mesh size. We assume that the mesh is quasi-uniform. We further assume that $\Gamma$ coincides with gridlines of $\mathcal{T}_{h}$. For a non-negative integer $r$ we let the space $W_{h}$ consist of continuous piecewise polynomials of degree at most $r+1$, i.e.

$$
W_{h}=\left\{q \in H_{0}^{1}(\Omega ; \Gamma):\left.q\right|_{K} \in P_{r+1}(K) \quad \text { for all } K \in \mathcal{T}_{h}\right\},
$$


where $P_{r}(K)$ denotes the space of polynomials of degree $r$ on $K$. The space $S_{h}$ is constructed from $W_{h}$ by setting

$$
S_{h}=\left.W_{h}\right|_{\Gamma},
$$

i.e. $S_{h}$ is the trace space of $W_{h}$. Let us recall that the quasi-uniformity of the mesh implies a number of inverse inequalities. In the discussion below we will use the fact that there is a constant $c$, independent of $h$, such that

$$
|\sigma|_{1 / 2+\theta} \leq c h^{-1 / 2}|\sigma|_{\theta} \quad \text { for all } \sigma \in S_{h} \text { and } \theta=0,1 / 2 .
$$

A well-known consequence of (4.2) and approximation properties is that the $L^{2}$ projection $Q_{h}: L^{2}(\Gamma) \mapsto S_{h}$ is uniformly bounded in $H_{0}^{1 / 2}(\Gamma)$ and $H^{-1 / 2}(\Gamma)$. Furthermore, for any $\sigma \in S_{h}$ the norm $|\sigma|_{-1 / 2}$ can equivalently be characterized by duality with respect to $S_{h}$, i.e. the two norms

$$
|\sigma|_{-1 / 2} \quad \text { and } \sup _{g \in S_{h}} \frac{\langle\sigma, g\rangle}{|g|_{1 / 2}}
$$

are equivalent on $S_{h}$, uniformly in $h$. Similarly, the two norms

$$
|g|_{1 / 2} \quad \text { and } \sup _{\sigma \in S_{h}} \frac{\langle g, \sigma\rangle}{|\sigma|_{-1 / 2}}
$$

are also equivalent on $S_{h}$.

Furthermore, it is established in [6] that for any $g \in S_{h}$

$$
\inf \left\{\|q\|_{1}: q \in W_{h}, T q=g\right\} \leq c|g|_{1 / 2},
$$

where the constant $c$ is independent of $g$ and $h$. Hence, we can conclude that the norms

$$
|\sigma|_{-1 / 2} \quad \text { and } \sup _{q \in W_{h}} \frac{\langle\sigma, T q\rangle}{\|q\|_{1}}
$$

are equivalent on $S_{h}$, uniformly in $h$.

The discrete problem corresponding to (2.5) is then to find $\left(p_{h}, \lambda_{h}\right) \in W_{h} \times S_{h}$ such that

$$
\begin{aligned}
a\left(p_{h}, q\right)+\left\langle\lambda_{h},\left.q\right|_{\Gamma}\right\rangle & =(f, q) \text { for all } q \in W_{h}, \\
\left\langle\left. p_{h}\right|_{\Gamma}, \sigma\right\rangle & =\langle g, \sigma\rangle \text { for all } \sigma \in S_{h} .
\end{aligned}
$$

This system has a unique solution. Furthermore, $p_{h} \in W_{h}$ satisfies $\left.p_{h}\right|_{\Gamma}=Q_{h} g$ and

$$
a\left(p_{h}, q\right)=(f, q) \text { for all } q \in W_{h}^{0},
$$

where $W_{h}^{0}=\left\{q \in W_{h}:\left.q\right|_{\Gamma}=0\right\}$. Hence, in this sense $p_{h}$ can be seen as a standard finite element approximation of $p$ derived from the weak formulation (2.4). The variable $\lambda_{h} \in S_{h}$ is an approximation of $-\partial p / \partial \boldsymbol{n}$ on $\Gamma$.

The discrete system may also be formulated in operator form by introducing the discrete coefficient operator $\mathcal{A}_{h}$. Let $\mathcal{A}_{h}: W_{h} \times S_{h} \mapsto W_{h} \times S_{h}$ be the $L^{2}$-symmetric, indefinite operator defined by

$$
\mathcal{A}_{h}=\left(\begin{array}{cc}
A_{h} & T_{h}^{*} \\
T_{h} & 0
\end{array}\right),
$$

where $T_{h}: W_{h} \mapsto S_{h}$ is the trace operator, $T_{h}^{*}: S_{h} \mapsto W_{h}$ its $L^{2}$-dual. Furthermore, $A_{h}: W_{h} \mapsto W_{h}$ is given by

$$
\left(A_{h} p, q\right)=a(p, q) \quad \text { for } p, q \in W_{h} .
$$


Hence, $A_{h}$ corresponds to an approximation of the elliptic operator $-\operatorname{div}(K \operatorname{grad} \cdot)$ with Neumann boundary conditions on $\Gamma$. The system (4.6) can now be written in the form

$$
\mathcal{A}_{h}\left(\begin{array}{c}
p_{h} \\
\lambda_{h}
\end{array}\right)=\left(\begin{array}{l}
f_{h} \\
g_{h}
\end{array}\right)
$$

where $f_{h} \in W_{h}$ and $g_{h} \in S_{h}$ are $L^{2}$-projections of $f$ and $g$.

Let the norm on $W_{h}$ be $\|\cdot\|_{1}$. The dual space $W_{h}^{*}$ is equal to $W_{h}$ as a set, but with norm

$$
\|p\|_{W_{h}^{*}}=\sup _{q \in W_{h}} \frac{(p, q)}{\|q\|_{1}} .
$$

Similarly, we let $|\cdot|_{-1 / 2}$ be the norm on $S_{h}$, while $S_{h}^{*}$ denotes this space equipped with the norm $|\cdot|_{1 / 2}$. The discrete elliptic operator $A_{h}$ has the property that the operator norms

$$
\left\|A_{h}\right\|_{\mathcal{L}\left(W_{h}, W_{h}^{*}\right)} \quad \text { and } \quad\left\|A_{h}^{-1}\right\|_{\mathcal{L}\left(W_{h}^{*}, W_{h}\right)}
$$

are bounded uniformly in $h$. Together with (4.5) above we derive by straightforward energy estimates that

$$
\left\|\mathcal{A}_{h}\right\|_{\mathcal{L}\left(X_{h}, X_{h}^{*}\right)} \quad \text { and } \quad\left\|\mathcal{A}_{h}^{-1}\right\|_{\mathcal{L}\left(X_{h}^{*}, X_{h}\right)} \quad \text { are bounded uniformly in } h .
$$

Here, $X_{h}$ and $X_{h}^{*}$ denote the product spaces $X_{h}=W_{h} \times S_{h}$ and $X_{h}^{*}=W_{h}^{*} \times S_{h}^{*}$. Note that the property (4.9) corresponds to the fact that the continuous operator $\mathcal{A}$ is an isomorphism from $H_{0}^{1}(\Omega ; \Gamma) \times H^{-1 / 2}(\Gamma)$ into $H_{0}^{1}(\Omega ; \Gamma)^{*} \times H_{0}^{1 / 2}(\Gamma)$.

As a consequence of the mapping property (4.9) for $\mathcal{A}_{h}$, an obvious choice of a preconditioner $\mathcal{B}_{h}$ is an $L^{2}$-symmetric, positive definite block diagonal operator

$$
\mathcal{B}_{h}=\left(\begin{array}{cc}
M_{h} & 0 \\
0 & N_{h}
\end{array}\right): W_{h} \times S_{h} \mapsto W_{h} \times S_{h}
$$

such that

$$
\left\|\mathcal{B}_{h}\right\|_{\mathcal{L}\left(X_{h}^{*}, X_{h}\right)} \quad \text { and } \quad\left\|\mathcal{B}_{h}^{-1}\right\|_{\mathcal{L}\left(X_{h}, X_{h}^{*}\right)} \quad \text { are bounded uniformly in } h .
$$

As in the continuous case we then obtain that the operator $\mathcal{B}_{h} \mathcal{A}_{h}$ is symmetric with respect to the inner product $\left(\mathcal{B}_{h}^{-1} \cdot, \cdot\right)$, with spectral condition number $\kappa\left(\mathcal{B}_{h} \mathcal{A}_{h}\right)$ independent of $h$.

4.1. Domain embedding preconditioners. Let $\left\{\mathcal{T}_{e, h}\right\}_{h \in(0,1]}$ be a family of triangulations of the extended domain $\Omega_{e}$ such that $\mathcal{T}_{h}$ is obtained by restricting $\mathcal{T}_{e, h}$ to $\Omega$. Let $W_{e, h} \subset H_{0}^{1}\left(\Omega_{e}\right)$ be the corresponding finite element space with continuous piecewise polynomials of degree at most $r+1$. The purpose of the rest of this section is to discuss how domain embedding can be utilized in order to construct the preconditioners $M_{h}$ and $N_{h}$. Recall that (4.10) implies that $M_{h}: W_{h} \mapsto W_{h}$ is required to be a uniform preconditioner for the discrete elliptic operator $A_{h}$, corresponding to Neumann boundary conditions on $\Gamma$, and that such operators can be constructed by a discrete analog of (3.3). We will therefore only discuss here the operator $N_{h}$. By (4.10) the operator $N_{h}: S_{h} \mapsto S_{h}$ needs to be constructed so that

$$
\left\|N_{h}\right\|_{\mathcal{L}\left(S_{h}^{*}, S_{h}\right)} \quad \text { and } \quad\left\|N_{h}^{-1}\right\|_{\mathcal{L}\left(S_{h}, S_{h}^{*}\right)} \text { are bounded uniformly in } h .
$$

In order to define a discrete analog of the operator $N$ discussed in $\S 3.2$ we need to discretize the problem (3.7). A natural space for discretizing $\boldsymbol{H}\left(\operatorname{div} ; \Omega_{e}\right)$ is the Raviart-Thomas space. For the non-negative integer $r$ associated with the space 
$W_{h}$ of continuous piecewise polynomials, the corresponding Raviart-Thomas space of index $r$ is given by

$$
\boldsymbol{V}_{h}=\left\{\boldsymbol{v} \in \boldsymbol{H}\left(\operatorname{div} ; \Omega_{e}\right):\left.\boldsymbol{v}\right|_{K} \in \boldsymbol{P}_{r}(K)+\boldsymbol{x} P_{r}(K) \text { for all } K \in \mathcal{T}_{e, h}\right\},
$$

where $P_{r}(K)$ denotes the space of polynomials of degree $r$ on $K$. A vector field in $\boldsymbol{V}_{h}$ is uniquely specified by giving its value at $r(r+1) / 2$ points in each triangle and the value of its normal component at $r+1$ points on each edge of the triangulation. We will also need the trace space of $\boldsymbol{V}_{h} \cdot \boldsymbol{n}$ on $\Gamma$, which is defined by:

$$
Z_{h}=\left\{\mu: \mu=(\boldsymbol{v} \cdot \boldsymbol{n})_{\Gamma} \text { for } \boldsymbol{v} \in \boldsymbol{V}_{h}\right\} .
$$

Hence, $Z_{h}$ is a space of discontinuous piecewise polynomials on $\Gamma$.

For a given $g \in S_{h}$ the problem (3.7) is replaced by: Find $\boldsymbol{u}_{h} \in \boldsymbol{V}_{h}$ such that

$$
\Lambda\left(\boldsymbol{u}_{h}, \boldsymbol{v}\right)=\left\langle g,(\boldsymbol{v} \cdot \boldsymbol{n})_{\Gamma}\right\rangle \quad \text { for all } \boldsymbol{v} \in \boldsymbol{V}_{h} .
$$

Recall that $Q_{h}: L^{2}(\Gamma) \mapsto S_{h}$ is the $L^{2}$-projection. Define $\bar{N}_{h}: S_{h} \mapsto S_{h}$ by

$$
\bar{N}_{h} g=Q_{h}\left(\boldsymbol{u}_{h} \cdot \boldsymbol{n}\right)_{\Gamma},
$$

where $\boldsymbol{u}_{h} \in \boldsymbol{V}_{h}$ solves (4.13). It follows directly from the trace inequality (3.10) that

$$
\left|\bar{N}_{h} g\right|_{-1 / 2}^{2} \leq c_{1}^{2} \Lambda\left(\boldsymbol{u}_{h}, \boldsymbol{u}_{h}\right)=c_{1}^{2}\left\langle g,\left(\boldsymbol{u}_{h} \cdot \boldsymbol{n}\right)_{\Gamma}\right\rangle \leq c_{1}^{2}|g|_{1 / 2}\left|\bar{N}_{h} g\right|_{-1 / 2}
$$

or

$$
\left|\bar{N}_{h} g\right|_{-1 / 2} \leq c_{1}^{2}|g|_{1 / 2} .
$$

Hence, the continuous bound (3.12) carries directly over to the discrete case.

Let $Q_{h}^{*}: L^{2}(\Gamma) \mapsto Z_{h}$ be the $L^{2}$-projection. The preconditioner $N_{h}: S_{h} \mapsto S_{h}$ is now given by

$$
N_{h}=\bar{N}_{h}+\alpha h^{-1} Q_{h}\left(I-Q_{h}^{*}\right)
$$

for some positive constant $\alpha$. It is straightforward to check that $N_{h}$ is $L^{2}$-symmetric and positive definite. Furthermore, since $Z_{h}$ contains piecewise constants,

$$
\left|\left(I-Q_{h}^{*}\right) g\right|_{0} \leq c h|g|_{1} \quad \text { for all } g \in S_{h},
$$

where $c$ is independent of $h$. Together with the inverse inequality (4.2) and the result (4.3), this shows that

$$
\begin{aligned}
\left|Q_{h}\left(I-Q_{h}^{*}\right) g\right|_{-1 / 2} & \leq c \sup _{\sigma \in S_{h}} \frac{\left\langle\left(I-Q_{h}^{*}\right) g, \sigma\right\rangle}{|\sigma|_{1 / 2}}=c \sup _{\sigma \in S_{h}} \frac{\left\langle\left(I-Q_{h}^{*}\right) g,\left(I-Q_{h}^{*}\right) \sigma\right\rangle}{|\sigma|_{1 / 2}} \\
& \leq c \sup _{\sigma \in S_{h}} \frac{\left|\left(I-Q_{h}^{*}\right) g\right|_{0}\left|\left(I-Q_{h}^{*}\right) \sigma\right|_{0}}{|\sigma|_{1 / 2}} \leq c h|g|_{1 / 2},
\end{aligned}
$$

where the constant $c$ is independent of $h$.

Together with (4.14) this implies that $N_{h} \in \mathcal{L}\left(S_{h}^{*}, S_{h}\right)$ is uniformly bounded. In fact, the following theorem shows that $N_{h}$ satisfies (4.11).

Theorem 4.1. The operator $N_{h}: S_{h} \mapsto S_{h}$ defined above is an $L^{2}$-symmetric, positive definite operator such that

$$
\left\|N_{h}\right\|_{\mathcal{L}\left(S_{h}^{*}, S_{h}\right)} \quad \text { and } \quad\left\|N_{h}^{-1}\right\|_{\mathcal{L}\left(S_{h}, S_{h}^{*}\right)}
$$

are bounded uniformly in $h$. 
Proof. It remains to bound $\left\|N_{h}^{-1}\right\|_{\mathcal{L}\left(S_{h}, S_{h}^{*}\right)}$. We shall need a discrete extension result corresponding to (3.13). The following extension result is proved in [19, Lemma 4.3].

Given $\mu \in Z_{h}$, there exists a $\boldsymbol{v} \in \boldsymbol{V}_{h}$ with $(\boldsymbol{v} \cdot \boldsymbol{n})_{\Gamma}=\mu$ and

$\|\boldsymbol{v}\|_{\text {div }} \leq c|\mu|_{-1 / 2}$ for some constant $c$ independent of $\mu$ and $h$.

In addition, we claim that $Q_{h}^{*}$ is stable in $H^{-1 / 2}(\Gamma)$, i.e. there is a constant $c$, independent of $h$, such that

$$
\left|Q_{h}^{*} \sigma\right|_{-1 / 2} \leq c|\sigma|_{-1 / 2} \text { for all } \sigma \in S_{h} .
$$

In order to see this, observe first that it follows from (4.2) and duality that

$$
|\sigma|_{0} \leq c h^{-1 / 2}|\sigma|_{-1 / 2} \quad \text { for all } \sigma \in S_{h}
$$

Therefore, we have

$$
\begin{aligned}
\left|Q_{h}^{*} \sigma\right|_{-1 / 2} & =\sup _{g \in H_{0}^{1 / 2}(\Gamma)} \frac{\left\langle Q_{h}^{*} \sigma, g\right\rangle}{|g|_{1 / 2}} \leq \sup _{g \in H_{0}^{1 / 2}(\Gamma)} \frac{\langle\sigma, g\rangle}{|g|_{1 / 2}}+\sup _{g \in H_{0}^{1 / 2}(\Gamma)} \frac{\left\langle\sigma,\left(I-Q_{h}^{*}\right) g\right\rangle}{|g|_{1 / 2}} \\
& \leq|\sigma|_{-1 / 2}+c h^{1 / 2}|\sigma|_{0} \leq c|\sigma|_{-1 / 2},
\end{aligned}
$$

and hence (4.18) is established.

In order to bound $|g|_{1 / 2}$ by $\left|N_{h} g\right|_{-1 / 2}$ we split the norm of $g$ into two parts. For a given $g \in S_{h}$ we have from (4.4) that

$$
|g|_{1 / 2}=\sup _{\sigma \in S_{h}} \frac{\langle g, \sigma\rangle}{|\sigma|_{-1 / 2}} \leq \sup _{\sigma \in S_{h}} \frac{\left\langle g, Q_{h}^{*} \sigma\right\rangle}{|\sigma|_{-1 / 2}}+\sup _{\sigma \in S_{h}} \frac{\left\langle\left(I-Q_{h}^{*}\right) g, \sigma\right\rangle}{|\sigma|_{-1 / 2}} .
$$

We will estimate each term on the right hand side of (4.20). For the first term we note that (4.17) and (4.18) imply that

$$
\sup _{\sigma \in S_{h}} \frac{\left\langle g, Q_{h}^{*} \sigma\right\rangle}{|\sigma|_{-1 / 2}} \leq c \sup _{\sigma \in S_{h}} \frac{\left\langle g, Q_{h}^{*} \sigma\right\rangle}{\left|Q_{h}^{*} \sigma\right|_{-1 / 2}} \leq c \sup _{\boldsymbol{v} \in \boldsymbol{V}_{h}} \frac{\left\langle g,(\boldsymbol{v} \cdot \boldsymbol{n})_{\Gamma}\right\rangle}{\|\boldsymbol{v}\|_{\text {div }}} .
$$

Hence, if we let $\boldsymbol{u}_{h} \in \boldsymbol{V}_{h}$ solve (4.13), then

$$
\sup _{\sigma \in S_{h}} \frac{\left\langle g, Q_{h}^{*} \sigma\right\rangle}{|\sigma|_{-1 / 2}} \leq c \sup _{\boldsymbol{v} \in \boldsymbol{V}_{h}} \frac{\Lambda\left(\boldsymbol{u}_{h}, \boldsymbol{v}\right)}{\|\boldsymbol{v}\|_{\text {div }}} \leq c \Lambda\left(\boldsymbol{u}_{h}, \boldsymbol{u}_{h}\right)^{1 / 2}=c\left\langle\bar{N}_{h} g, g\right\rangle^{1 / 2} .
$$

On the other hand, by using (4.19) and the Cauchy-Schwarz inequality we obtain for the second term

$$
\sup _{\sigma \in S_{h}} \frac{\left\langle\left(I-Q_{h}^{*}\right) g, \sigma\right\rangle}{|\sigma|_{-1 / 2}} \leq \sup _{\sigma \in S_{h}} \frac{\left|\left(I-Q_{h}^{*}\right) g\right|_{0}|\sigma|_{0}}{|\sigma|_{-1 / 2}} \leq c\left\langle h^{-1} Q_{h}\left(I-Q_{h}^{*}\right) g, g\right\rangle^{1 / 2} .
$$

By combining this with (4.20) and (4.21) we now have

$$
\begin{aligned}
|g|_{1 / 2} & \leq c\left(\left\langle\bar{N}_{h} g, g\right\rangle^{1 / 2}+\left\langle h^{-1} Q_{h}\left(I-Q_{h}^{*}\right) g, g\right\rangle^{1 / 2}\right) \\
& \leq c\left\langle N_{h} g, g\right\rangle^{1 / 2} \leq c\left|N_{h} g\right|_{-1 / 2}^{1 / 2}|g|_{1 / 2}^{1 / 2},
\end{aligned}
$$

and hence $\left\|N_{h}^{-1}\right\|_{\mathcal{L}\left(S_{h}, S_{h}^{*}\right)}$ is bounded uniformly in $h$.

Instead of solving the equation (4.13) exactly, one can use a suitable preconditioner. Let $\boldsymbol{\Lambda}_{h}: \boldsymbol{V}_{h} \mapsto \boldsymbol{V}_{h}$ be the operator associated with the bilinear form $\Lambda$, i.e.

$$
\left(\boldsymbol{\Lambda}_{h} \boldsymbol{u}, \boldsymbol{v}\right)_{e}=\Lambda(\boldsymbol{u}, \boldsymbol{v}) \quad \text { for all } \boldsymbol{u}, \boldsymbol{v} \in \boldsymbol{V}_{h}
$$


Furthermore, let $\boldsymbol{G}_{h}: S_{h} \mapsto \boldsymbol{V}_{h}$ be defined by

$$
\left(\boldsymbol{G}_{h} g, \boldsymbol{v}\right)_{e}=\left\langle g,(\boldsymbol{v} \cdot \boldsymbol{n})_{\Gamma}\right\rangle \quad \text { for } g \in S_{h}, \boldsymbol{v} \in \boldsymbol{V}_{h} .
$$

Hence, we have

$$
\left\langle\bar{N}_{h} g, \sigma\right\rangle=\left(\boldsymbol{\Lambda}_{h}^{-1} \boldsymbol{G}_{h} g, \boldsymbol{G}_{h} \sigma\right)_{e} \quad \text { for all } g, \sigma \in S_{h}
$$

Assume now that $\boldsymbol{\Theta}_{h}: \boldsymbol{V}_{h} \mapsto \boldsymbol{V}_{h}$ is a uniform preconditioner for $\boldsymbol{\Lambda}_{h}$, i.e. the bilinear forms

$$
\left(\boldsymbol{\Lambda}_{h}^{-1} \boldsymbol{v}, \boldsymbol{v}\right)_{e} \quad \text { and } \quad\left(\boldsymbol{\Theta}_{h} \boldsymbol{v}, \boldsymbol{v}\right)_{e} \quad \text { are uniformly equivalent }
$$

with respect to $h$ on $\boldsymbol{V}_{h}$. Define an operator $\widehat{N}_{h}: S_{h} \mapsto S_{h}$ by

$$
\widehat{N}_{h} g=Q_{h}\left(\widehat{\boldsymbol{u}}_{h} \cdot \boldsymbol{n}\right)_{\Gamma},
$$

where $\widehat{\boldsymbol{u}}_{h}=\boldsymbol{\Theta}_{h} \boldsymbol{G}_{h} g \in \boldsymbol{V}_{h}$. Then

$$
\left\langle\widehat{N}_{h} g, \sigma\right\rangle=\left(\boldsymbol{\Theta}_{h} \boldsymbol{G}_{h} g, \boldsymbol{G}_{h} \sigma\right)_{e} \quad \text { for all } g, \sigma \in S_{h},
$$

and as a consequence of (4.22) and (4.23) the bilinear forms

$$
\left\langle\bar{N}_{h} g, g\right\rangle \text { and }\left\langle\widehat{N}_{h} g, g\right\rangle \text { are uniformly equivalent in } h \text {. }
$$

Therefore, the operator $N_{h}: S_{h} \mapsto S_{h}$ given by

$$
N_{h}=\widehat{N}_{h}+\alpha h^{-1} Q_{h}\left(I-Q_{h}^{*}\right)
$$

will satisfy the mapping property (4.11).

We have therefore seen that a uniform preconditioner $\boldsymbol{\Theta}_{h}$ for the operator $\boldsymbol{\Lambda}_{h}$ associated with the $\boldsymbol{H}$ (div)-inner product $\Lambda$, defined on the extended domain $\Omega_{e}$, can be used to construct a suitable preconditioner $N_{h}$. Preconditioners for $\boldsymbol{\Lambda}_{h}$ are discussed in [2], [8] and [21]. We should note here that the differential operator $\boldsymbol{\Lambda}$, given by (3.6), has the property that it acts like a second-order elliptic operator on gradient fields, while $\boldsymbol{\Lambda}$ coincides with the identity on curl fields. Hence, $\boldsymbol{\Lambda}$ is not an elliptic operator and, as a consequence, the construction of, for example, multilevel preconditioners for the corresponding discrete operator $\boldsymbol{\Lambda}_{h}$ does not appear to be straightforward. However, it is established in [2] that a standard multigrid Vcycle operator, with a proper smoothing operator, will in fact lead to a uniform preconditioner.

\section{Numerical EXAmples}

In this section we will present some numerical examples where we use the preconditioner described in the previous sections. In these examples $\Omega$ will be the $\mathrm{L}$-shaped domain shown in Figure 2, where $\partial \Omega=\Gamma \cup \Upsilon$ and $\Upsilon=\Upsilon_{1} \cup \Upsilon_{2} \cup \Upsilon_{3} \cup \Upsilon_{4}$. The extended domain $\Omega_{e}$ is equal to the unit square. The triangulation of $\Omega$ and $\Omega_{e}$ is obtained by first dividing the domain into $h \times h$ sized squares, and then dividing each square into two triangles by using the negatively sloped diagonal. The finite element spaces $W_{h}, W_{e, h}$ and $\boldsymbol{V}_{h}$ are chosen with $r=0$. Hence, the spaces $W_{h}$ and $W_{e, h}$ consist of continuous piecewise linear functions. The space $\boldsymbol{V}_{h}$ is the lowest order Raviart-Thomas space, i.e. $\boldsymbol{V}_{h}$ consists of vector functions with continuous normal component on the edges of the triangulation, which on each element have the form $\boldsymbol{a}+b \boldsymbol{x}$ for $\boldsymbol{a} \in \mathbb{R}^{2}$ and $b \in \mathbb{R}$. The trace space of $W_{h}$ on $\Gamma, S_{h}$, consists of one dimensional continuous piecewise linear functions, while $Z_{h}$ is the space of piecewise constants on $\Gamma$. A version of the minimum residual method [17] is used to solve the linear systems. The iterations are terminated when the residual of the 

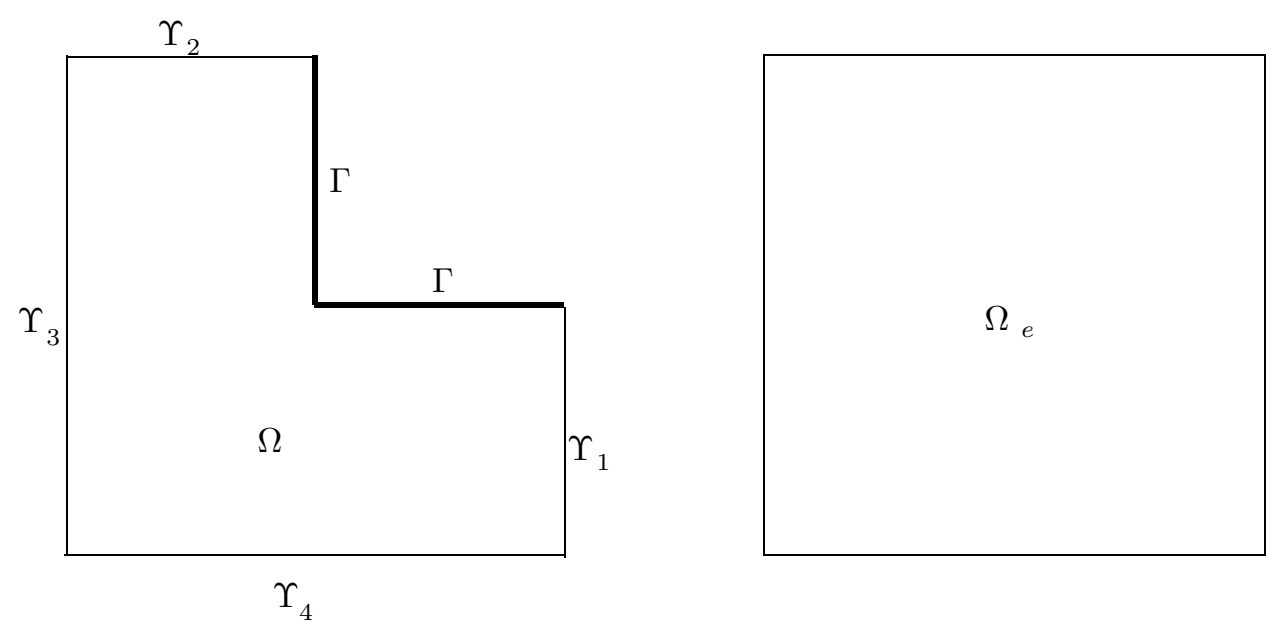

Figure 2. The domain used in the numerical examples in $\S 5$. The extended domain $\Omega_{e}$ is the unit square.

preconditioned system is reduced by a factor $10^{-5}$ in the norm induced by the inner product $\left(\mathcal{B}_{h}^{-1} \cdot, \cdot\right)$.

The domain embedding preconditioner $M_{h}: W_{h} \mapsto W_{h}$ is chosen of the form indicated by (3.3). More precisely,

$$
M_{h}=R_{h} B_{e, h} E_{h}
$$

where $R_{h}: W_{e, h} \mapsto W_{h}$ is the restriction operator, and $E_{h}: W_{h} \mapsto W_{e, h}$ is the $L^{2}$-adjoint operator. Furthermore, $B_{e, h}: W_{e, h} \mapsto W_{e, h}$ is a preconditioner for the operator associated with an extension of the bilinear form (2.3). In the examples below $B_{e, h}$ is a multigrid V-cycle operator with an SSOR-smoother.

The preconditioner $N_{h}: S_{h} \mapsto S_{h}$ will be of the form (4.24) with $\alpha=3.0$. In particular, the preconditioner $\boldsymbol{\Theta}_{h}: \boldsymbol{V}_{h} \mapsto \boldsymbol{V}_{h}$ for the operator $\boldsymbol{\Lambda}_{h}$ is chosen as the $\mathrm{V}$-cycle operator described in [2], with a multiplicative Schwarz operator as a smoother.

Example 5.1. In the first example we solve equation (4.6) with $K(\boldsymbol{x})$ equal to the identity matrix, $f(\boldsymbol{x})=1$ and $g(\boldsymbol{x})=\left(1-\left(2 x_{1}-1\right)^{2}\right)\left(1-\left(2 x_{2}-1\right)^{2}\right)$.

The condition number $\kappa\left(\mathcal{B}_{h} \mathcal{A}_{h}\right)$ and the required number of iterations are displayed in Table 1. Here, the condition numbers are estimated by a standard technique from the conjugate gradient method applied to the normal equations. The results clearly seem to confirm our theory above, which predicts that both the condition number and the number of iterations are bounded from above as $h \rightarrow 0$.

TABLE 1. The condition number for $\mathcal{B}_{h} \mathcal{A}_{h}$ and the number of iterations used when solving the Poisson problem in Example 5.1

\begin{tabular}{|c|c|c|c|c|c|}
\hline$h$ & $1 / 8$ & $1 / 16$ & $1 / 32$ & $1 / 64$ & $1 / 128$ \\
\hline$\kappa\left(\mathcal{B}_{h} \mathcal{A}_{h}\right)$ & 2.24 & 2.28 & 2.34 & 2.39 & 2.43 \\
\hline no. of iterations & 10 & 13 & 15 & 15 & 15 \\
\hline
\end{tabular}


5.1. Stokes problem. We will next try to adopt the domain embedding preconditioning technique to the Stokes problem with Dirichlet boundary conditions. The computations will be done on the $\mathrm{L}$-shaped domain in Figure 2. The Stokes equations model the flow of a (very) viscous fluid. The steady state problem, with $\boldsymbol{u}$ denoting the velocity and $p$ the pressure, is given by

$$
\begin{array}{rll}
-\Delta \boldsymbol{u}+\operatorname{grad} p & =\boldsymbol{f} & \text { in } \Omega, \\
\operatorname{div} \boldsymbol{u} & =0 & \text { in } \Omega .
\end{array}
$$

We will assume that $\Gamma$ is an impermeable part of the boundary. Hence, the Dirichlet boundary conditions are of the form

$$
\begin{array}{ll}
\boldsymbol{u}=\mathbf{0} & \text { on } \Gamma, \\
\boldsymbol{u}=\boldsymbol{g} & \text { on } \Upsilon .
\end{array}
$$

Here, the Dirichlet data $\boldsymbol{g} \in \boldsymbol{H}_{\mathbf{0}}^{1 / 2}(\Upsilon)$ satisfies the compatibility condition

$$
\int_{\Upsilon} \boldsymbol{g} \cdot \boldsymbol{n} d s=0
$$

In order to state a weak formulation of the problem we define the function spaces

$$
\begin{aligned}
\boldsymbol{H}_{\boldsymbol{g}}^{1}(\Omega ; \Gamma) & =\left\{\boldsymbol{v} \in\left(H^{1}(\Omega)\right)^{2}:\left.\boldsymbol{v}\right|_{\Upsilon}=\boldsymbol{g} \in \boldsymbol{H}_{\mathbf{0}}^{1 / 2}(\Upsilon)\right\} \\
L_{0}^{2}(\Omega) & =\left\{q \in L^{2}(\Omega): \int_{\Omega} q d \boldsymbol{x}=0\right\} .
\end{aligned}
$$

Introducing Lagrange multipliers $\boldsymbol{\lambda} \in \boldsymbol{H}^{-1 / 2}(\Gamma)=\left(H^{-1 / 2}(\Gamma)\right)^{2}$ to transform the essential boundary conditions on $\Gamma$ into natural conditions, we obtain the weak formulation: Find $(\boldsymbol{u}, \boldsymbol{\lambda}, p) \in \boldsymbol{H}_{\boldsymbol{g}}^{1}(\Omega ; \Gamma) \times \boldsymbol{H}^{-1 / 2}(\Gamma) \times L_{0}^{2}(\Omega)$ such that

$$
\begin{aligned}
(\operatorname{grad} \boldsymbol{u}, \operatorname{grad} \boldsymbol{v})+\left\langle\boldsymbol{\lambda},\left.\boldsymbol{v}\right|_{\Gamma}\right\rangle-(p, \operatorname{div} \boldsymbol{v}) & =(\boldsymbol{f}, \boldsymbol{v}) & & \text { for all } \boldsymbol{v} \in \boldsymbol{H}_{\mathbf{0}}^{1}(\Omega ; \Gamma), \\
\left\langle\left.\boldsymbol{u}\right|_{\Gamma}, \boldsymbol{\sigma}\right\rangle & =0 & & \text { for all } \boldsymbol{\sigma} \in \boldsymbol{H}^{-1 / 2}(\Gamma), \\
-(\operatorname{div} \boldsymbol{u}, q) & =0 & & \text { for all } q \in L_{0}^{2}(\Omega) .
\end{aligned}
$$

For this system the Lagrange multiplier $\boldsymbol{\lambda}$ equals $-\partial \boldsymbol{u} / \partial \boldsymbol{n}+p \boldsymbol{n}$ on $\Gamma$. The forcing function $\boldsymbol{f}$ is assumed to be in $\boldsymbol{H}_{\mathbf{0}}^{1}(\Omega ; \Gamma)^{*}$, i.e. the dual space of $\boldsymbol{H}_{\mathbf{0}}^{1}(\Omega ; \Gamma)$ with respect to the $L^{2}$-inner product.

The system (5.2) can be written in operator form with a coefficient operator $\mathcal{A}$ given by

$$
\mathcal{A}=\left(\begin{array}{ccc}
-\Delta & \boldsymbol{T}^{*} & \operatorname{grad} \\
\boldsymbol{T} & 0 & \mathbf{0} \\
-\operatorname{div} & 0 & 0
\end{array}\right),
$$

where $\boldsymbol{T}$ is just a vector version of the trace operator and $\boldsymbol{T}^{*}$ its dual. Since our interest in the mapping properties of the coefficient operator is motivated by the desire to use iterative methods, it is sufficient to consider the operator $\mathcal{A}$ for functions $\boldsymbol{u}$ which satisfy homogeneous boundary conditions, i.e. $\boldsymbol{g}=\mathbf{0}$. This is because differences of two solutions in the iteration will have this property. Therefore, we consider

$$
\mathcal{A}: \boldsymbol{H}_{\mathbf{0}}^{1}(\Omega ; \Gamma) \times \boldsymbol{H}^{-1 / 2}(\Gamma) \times L_{0}^{2}(\Omega) \mapsto \boldsymbol{H}_{\mathbf{0}}^{1}(\Omega ; \Gamma)^{*} \times \boldsymbol{H}_{\mathbf{0}}^{1 / 2}(\Gamma) \times L_{0}^{2}(\Omega) .
$$


The operator $\mathcal{A}$ is an isomorphism on these spaces. As a consequence, a preconditioner $\mathcal{B}$ should be a block diagonal isomorphism mapping

$$
\boldsymbol{H}_{\mathbf{0}}^{1}(\Omega ; \Gamma)^{*} \times \boldsymbol{H}_{\mathbf{0}}^{1 / 2}(\Gamma) \times L_{0}^{2}(\Omega) \quad \text { onto } \quad \boldsymbol{H}_{\mathbf{0}}^{1}(\Omega ; \Gamma) \times \boldsymbol{H}^{-1 / 2}(\Gamma) \times L_{0}^{2}(\Omega) .
$$

Hence, if

$$
\mathcal{B}=\operatorname{diag}(\boldsymbol{M}, \beta \boldsymbol{N}, \gamma I),
$$

for some positive constants $\beta$ and $\gamma$, then the requirements on $\boldsymbol{M}, \boldsymbol{M}: \boldsymbol{H}_{\mathbf{0}}^{1}(\Omega ; \Gamma)^{*} \mapsto$ $\boldsymbol{H}_{\mathbf{0}}^{1}(\Omega ; \Gamma)$, and $\boldsymbol{N}, \boldsymbol{N}: \boldsymbol{H}_{\mathbf{0}}^{1 / 2}(\Gamma) \mapsto \boldsymbol{H}^{-1 / 2}(\Gamma)$, are simply vector versions of the corresponding requirements on the operators which were used to build the preconditioner for the Lagrange multiplier system (2.6) associated with the scalar problem (1.1).

Next, we consider a finite element discretization of the system (5.2). We assume that the domains $\Omega$ and $\Omega_{e}$ are triangulated as described above. Furthermore, the function $\boldsymbol{g}$ defined on $\Upsilon$ is assumed to be piecewise linear with respect to this triangulation (otherwise, replace $\boldsymbol{g}$ by its piecewise linear interpolant). We will use the space $\boldsymbol{U}_{\boldsymbol{g}, h} \subset \boldsymbol{H}_{\boldsymbol{g}}^{1}(\Omega ; \Gamma)$, consisting of continuous piecewise linear vector functions plus cubic bubble functions on each triangle. The finite element space $\boldsymbol{S}_{h} \subset \boldsymbol{H}_{\mathbf{0}}^{1 / 2}(\Gamma)$ is constructed by setting $\boldsymbol{S}_{h}=\left.\boldsymbol{U}_{\boldsymbol{g}, h}\right|_{\Gamma}$. Since the bubble functions are zero on the edges of the triangles, the space $\boldsymbol{S}_{h}$ will be equal to a vector version of the space $S_{h}$ used in Example 5.1 above. Finally, we use $W_{h} \subset L_{0}^{2}(\Omega)$ consisting of continuous piecewise linear functions to discretize the pressure.

The finite element method is now defined from the weak formulation (5.2) by replacing the spaces $\boldsymbol{H}_{\boldsymbol{g}}^{1}(\Omega ; \Gamma), \boldsymbol{H}_{\mathbf{0}}^{1 / 2}(\Gamma)$ and $L_{0}^{2}(\Omega)$ by the subspaces $\boldsymbol{U}_{\boldsymbol{g}, h}, \boldsymbol{S}_{h}$ and $W_{h}$, respectively. It is well-known that the pair of spaces $\left(\boldsymbol{U}_{\boldsymbol{g}, h}, W_{h}\right)$ satisfies the Babuška-Brezzi condition; cf. [1] or [7].

We will not perform a detailed analysis of the discrete system in this case. However, motivated by the discussion of the continuous problem above, we will propose a block diagonal preconditioner, and report the results of some numerical experiments.

The discrete coefficient operator $\mathcal{A}_{h}$ will be of the form

$$
\mathcal{A}_{h}=\left(\begin{array}{ccc}
-\boldsymbol{\Delta}_{h} & \boldsymbol{T}_{h}^{*} & \operatorname{grad}_{h} \\
\boldsymbol{T}_{h} & \mathbf{0} & \mathbf{0} \\
-\operatorname{div}_{h} & 0 & 0
\end{array}\right): \boldsymbol{U}_{\mathbf{0}, h} \times \boldsymbol{S}_{h} \times W_{h} \mapsto \boldsymbol{U}_{\mathbf{0}, h} \times \boldsymbol{S}_{h} \times W_{h} .
$$

Here, each block of the operator is defined implicitly by the discrete system. Based on the discussion of the continuous problem above, we consider a preconditioner $\mathcal{B}_{h}$ of the form

$$
\mathcal{B}_{h}=\operatorname{diag}\left(\boldsymbol{M}_{h}, \beta \boldsymbol{N}_{h}, \gamma I_{h}\right) .
$$

The operator $\boldsymbol{N}_{h}$, defined on $\boldsymbol{S}_{h}=\left(S_{h}\right)^{2}$, is a vector version of the operator $N_{h}$ used in Example 5.1. In the space $\boldsymbol{U}_{\mathbf{0}, h}$, the subspace spanned by the bubble functions is orthogonal to the space of continuous piecewise linear functions with respect to the Dirichlet form. Therefore, the operator $\boldsymbol{M}_{h}$ is constructed from a diagonal operator corresponding to the space spanned by the bubble functions, and by two copies of the scalar operator $M_{h}$ used above. Furthermore, the perturbation of the identity operator on $W_{h}$, corresponding to a lumping procedure, is introduced in order to avoid the inversion of the mass matrix with respect to $W_{h}$. 
TABLE 2. The condition number for $\mathcal{B}_{h} \mathcal{A}_{h}$ and the number of iterations used when solving the Stokes problem in Example 5.2.

\begin{tabular}{|c|c|c|c|c|c|}
\hline$h$ & $1 / 8$ & $1 / 16$ & $1 / 32$ & $1 / 64$ & $1 / 128$ \\
\hline$\kappa\left(\mathcal{B}_{h} \mathcal{A}_{h}\right)$ & 14.95 & 15.60 & 16.04 & 16.34 & 16.50 \\
\hline no. of iterations & 45 & 54 & 58 & 60 & 62 \\
\hline
\end{tabular}

Example 5.2. We will consider the case where the fluid flows into the domain through the $\Upsilon_{2}$ boundary and leaves the domain through the $\Upsilon_{1}$ boundary. The other boundaries will be impermeable. Hence, we chose $\boldsymbol{g} \mid \Upsilon_{1}=\left(x_{2}\left(0.5-x_{2}\right), 0\right)$, $\left.\boldsymbol{g}\right|_{\Upsilon_{2}}=\left(0,-x_{1}\left(0.5-x_{1}\right)\right)$ and $\left.\boldsymbol{g}\right|_{\Upsilon_{3}}=\left.\boldsymbol{g}\right|_{\Upsilon_{4}}=\mathbf{0}$. Furthermore, we used $\boldsymbol{f}=\mathbf{1}$. The parameters involved in the preconditioner (5.4) were chosen as $\beta=\gamma=8.0$. The condition numbers $\kappa\left(\mathcal{B}_{h} \mathcal{A}_{h}\right)$ and the required number of iterations for the minimum residual method are displayed in Table 2. We observe that the numbers are considerably higher than for the Poisson problem, but they still appear to be bounded from above independently of $h$.

\section{REFERENCES}

1. D.N. Arnold, F. Brezzi, and M. Fortin, A stable finite element method for the Stokes equation, Calcolo, 21:337-344, 1984. MR 86m:65136

2. D.N. Arnold, R.S. Falk, and R. Winther, Preconditioning in H(div) and applications, Math. Comp., 66:957-984, 1997. MR 97i:65177

3. G.P. Astrakhantsev, Methods of fictitious domains for a second order elliptic equation with natural boundary conditions, USSR Computational Math. and Math. Phys., 18:114-121, 1978. MR 57:8066

4. I. Babuška, The finite element method with Lagrangian multipliers, Numer. Math. 20:179182, 1973. MR 50:11806

5. J.H. Bramble, The Lagrange multiplier method for the Dirichlet problem, Math. Comp., 37:111, 1981. MR 83h:65119

6. J.H. Bramble, J.E. Pasciak, and A.H. Schatz, The construction of preconditioners for elliptic problems by substructuring I, Math. Comp., 47:103-134, 1986. MR 87m:65174

7. F. Brezzi and M. Fortin, Mixed and Hybrid Finite Element Methods, Springer-Verlag, 1991. MR 92d:65187

8. Z. Cai, C.I. Goldstein, and J.E. Pasciak, Multilevel iteration for mixed finite element systems with penalty, SIAM J. Sci. Comput., 14:1072-1088, 1993. MR 94h:65116

9. V. Girault and P.-A. Raviart, Finite Element Methods for Navier-Stokes Equations, SpringerVerlag, 1986. MR 88b:65129

10. R. Glowinski, T.-W. Pan, and J.Périaux, A fictitious domain method for Dirichlet problem and applications, Computer Methods in Applied Mechanics and Engineering, 111:283-303, 1994. MR 94k:65158

11. R. Glowinski, T.-W. Pan, and J.Périaux, On a domain embedding method for flow around moving rigid bodies, to appear in Bjørstad et. al., editor, Ninth International Symposium on Domain Decomposition Methods for Partial Differential Equations, Bergen, 1996.

12. W. Hackbusch, Iterative Solution of Large Sparse Systems of Equations, Springer-Verlag, 1994. MR 94k:65002

13. G.I. Marchuk, Y.A. Kuznetsov, and A.M. Matsokin, Fictitious domain and domain decomposition methods, Sov. Jour. Numer. Anal. Math. Modelling, 1:3-35, 1986. MR 88d:65061

14. S.V. Nepomnyaschikh, Decomposition and fictitious domains methods for elliptic boundary value problems, in Keyes et. al., editor, Fifth International Symposium on Domain Decomposition Methods for Partial Differential Equations, Philadelphia, 1992, pages 62-72. SIAM. MR 93i: 65116

15. S.V. Nepomnyaschikh, Mesh theorems on traces, normalizations of function traces and their inversion, Sov. J. Numer. Anal. Math. Model., 6(3):223-242, 1991. MR 93h:65148 
16. S.V. Nepomnyaschikh, Method of splitting into subspaces for solving elliptic boundary value problems in complex form domain, Sov. J. Numer. Anal. Math. Model., 6(2):151-168, 1991. MR 92i:65187

17. C.C. Paige and M.A. Saunders, Solution of sparse indefinite systems of linear equations, SIAM J. Numer. Anal., 12:617-629, 1975. MR 52:4595

18. T. Rossi, Fictitious domain methods with seperable preconditioners, Ph.D. thesis, University of Jyväskylä, Depertment of Mathematics, 1995.

19. T. Rusten, P.S. Vassilevski, and R. Winther, Domain embedding preconditioners for mixed systems, preprint, to appear in Numer. Lin Alg. Appl.

20. P.S. Vassilevski, On some applications of the $h^{\sigma}$-stable wavelet-like hierarchical finite element space decompositions, in Proceedings of the Conference on the Mathematics of Finite Elements and Applications, MAFELAP 1996, held June 25-28, 1996, Brunel University, London, UK., to be published by Wiley.

21. P.S. Vassilevski and J. Wang, Multilevel iterative methods for mixed finite element discretizations of elliptic problems, Numer. Math., 63:503-520, 1992. MR 93j:65187

SinteF, P. O. Box 124 Blindern, N-0314 Oslo, Norway

E-mail address: Einar.Haug@math.sintef .no

Department of Informatics, University of Oslo, P. O. Box 1080 Blindern, N-0316 OSLO, NORWAY

E-mail address: Ragnar.Winther@ifi.uio.no 